\title{
Untranslated Region SNP
}

National Cancer Institute

\section{Source}

National Cancer Institute. Untranslated Region SNP. NCI Thesaurus. Code C45392.

Single Nucleotide Polymorphism in UT R Sequences consists of a variation at an appreciable frequency between individuals of a single interbreeding population of a single nucleotide, due to base substitution, at an equivalent location within the sequence region at the $5^{\prime}$ end (preceding the initiation codon) or at the $3^{\prime}$ end (following the stop codon) of a messenger RNA that are not translated into protein and may contain transcription and translation regulating sequences. 